\title{
The influence of hypoglycemia on cerebral blood flow in cases of hypoglycemic coma
}

\author{
Yuzo Terakawa, N aohiro Tsuyuguchi, Akinori Yamamura ${ }^{1}$, Toshio N akagawa \\ Department of Neurosurgery, Osaka City University Graduate School of Medicine, Osaka, ${ }^{1}$ Department of Neurosurgery, Shinsapporo \\ Neurosurgical Hospital, Hokkaido, Japan
}

Address for correspondence:

Dr. Yuzo Terakawa,

Department of Neurosurgery,

O saka City University Graduate

School of M edicine 1-4-3

Asahi-machi, Abeno-ku, O saka

545-8585, Japan.

E-mail: terakawa@

msic.med.osaka-cu.ac.jp

DOI: $10.4103 / 0028-3886.48814$

\begin{abstract}
The authors report two cases of hypoglycemic coma in which both diffusion-weighted imaging (DWI) and single photon emission computed tomography (SPECT) were used. A 74 -year-old woman (case 1) presented with deep coma associated with hypoglycemia (blood glucose level: $20 \mathrm{mg} / \mathrm{dl}$ ), and DWI on admission revealed extensive hyperintensity lesions. SPECT obtained on the second hospital day revealed diffuse hyperperfusion and a follow-up SPECT on the eighth hospital day showed relative hypoperfusion. An 89- year- old woman (case 2) presented with coma associated with hypoglycemia (blood glucose level: $45 \mathrm{mg} / \mathrm{dl}$ ), and DWI showed diffuse hyperintensity lesions in both hemispheres. SPECT obtained on the ninth hospital day showed relative hypoperfusion compared to the first scan obtained on the third hospital day. Although the blood glucose level was rapidly corrected, the level of consciousness remained unchanged in both cases. Our results suggest that cerebral blood flow may increase even after glucose correction and that relative hypoperfusion could occur in the subacute stage of hypoglycemic coma.
\end{abstract}

Key words: Cerebral blood flow, diffusion-weighted imaging, hypoglycemia, hypoglycemic coma, single photon emission computed tomography

\section{Introduction}

It is well known that hypoglycemia leads to various neurological symptoms, ranging from reversible focal deficits to irreversible profound coma and death. However, changes of cerebral blood flow (CBF) in hypoglycemic coma in human patients have received scant attention. We report two cases of hypoglycemic coma in which both diffusion-weighted imaging (DWI) and single photon emission computed tomography (SPECT) were used and discuss the possible influence of hypoglycemia on CBF in hypoglycemic coma in conjunction with a review of the literature.

\section{Case Reports}

\section{Case 1}

A 74-year-old woman with a history of diabetes mellitus controlled with oral medication was admitted to our hospital after being found unconscious at home. Her family noticed that the patient had been drowsy the day before admission. On admission, she had slight hypertension (blood pressure: 158/80 $\mathrm{mmHg}$ ), regular heart rate, and irregular respiration but no hypoxia. Neurologically, the patient was in a deep coma (Glasgow Coma Scale 5) with nonreactive pinpoint pupils. Oculocephalic reflex was intact and Babinski reflex was positive bilaterally. The patient showed tetraplegia with decerebral posturing. The DWI obtained immediately after admission revealed bilateral symmetrical hyperintensity lesions in the middle cerebral peduncles, temporoparietal cortex, corona radiate, and the splenium of the corpus callosum [Figure 1]. Blood glucose level on admission was $20 \mathrm{mg} / \mathrm{dl}$ ( $1.1 \mathrm{mmol} / \mathrm{L})$. Although her blood glucose level was rapidly corrected to within the normal range by intravenous administration of glucose, her level of consciousness remained unchanged. SPECT using ${ }^{99 m}$ Tc-ethyl cysteinate dimer (ECD) obtained on the 

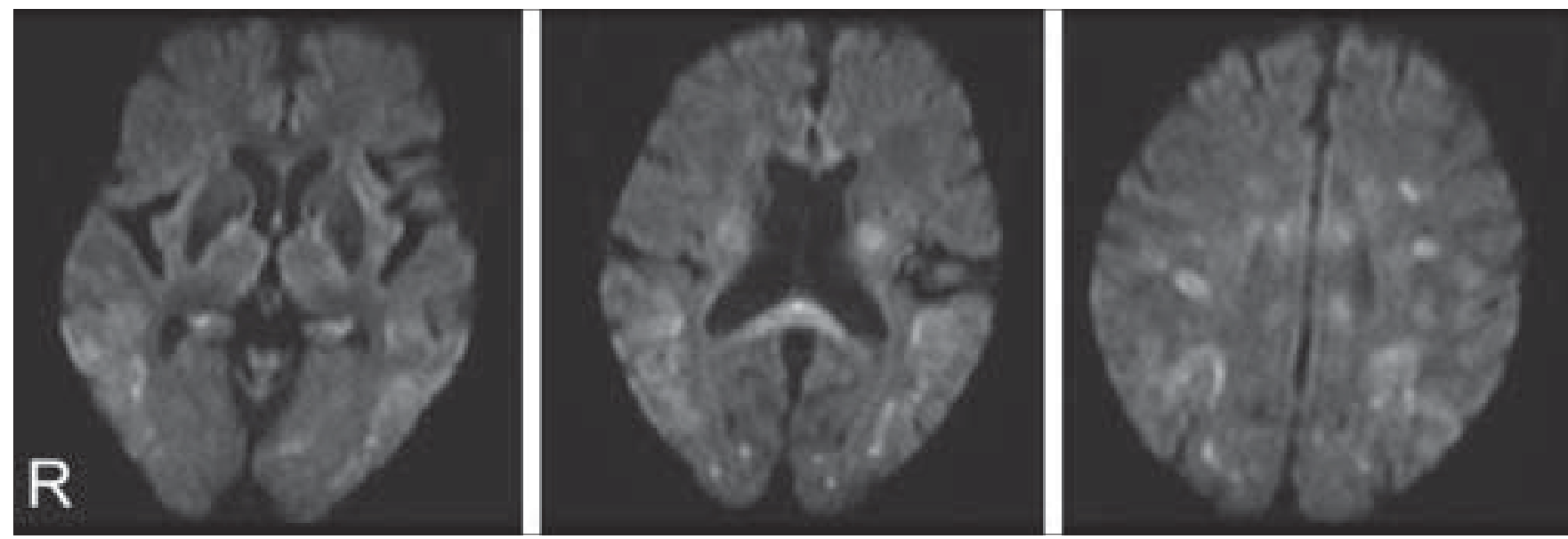

Figure 1: Diffusion-weighted images obtained immediately after admission showing multiple hyperintensity legions in the middle cerebral peduncles, temporoparietal cortex, corona radiate, and the splenium of the corpus callosum

following day revealed diffuse hyperperfusion in both hemispheres, especially in the bilateral temporoparietal regions. The second SPECT obtained on the eighth hospital day showed relative hypoperfusion compared to the first image [Figure 2].

\section{Case 2}

An 89-year-old woman with medically controlled diabetes mellitus and hypertension was admitted to our hospital for disturbance of consciousness. On admission, the patient had slight hypertension (blood pressure: $150 / 70 \mathrm{mmHg}$ ) but otherwise normal vital signs. Neurological examination showed the patient was in a coma (Glasgow Coma Scale 6) and tetraplegic. Babinski reflex was positive bilaterally. The DWI obtained revealed bilateral hyperintensity lesions in the temporoparietal cortex (more pronounced on the right side), internal capsules, deep white matter at the level of the centrum semiovale, and the splenium of the corpus callosum [Figure 3]. The laboratory data on admission showed that her blood glucose level was $45 \mathrm{mg} / \mathrm{dl}(2.5 \mathrm{mmol} / \mathrm{L})$. Despite glucose infusion, her level of consciousness did not improve. SPECT using 99mTc-hexamethyl propylene amine oxime (HMPAO) obtained two days after admission revealed relative hyperperfusion in the temporoparietal region. The follow-up SPECT obtained on the ninth hospital day showed relative hypoperfusion compared to the first scan except for the right temporoparietal region corresponding to the hyperintensity lesion on DWI [Figure 4].

\section{Discussion}

We reported two cases of hypoglycemic coma with repeat SPECT obtained both in the acute and subacute stages of hypoglycemic coma, and demonstrated changes in CBF associated with hypoglycemic coma. To our knowledge, there have been few previous reports

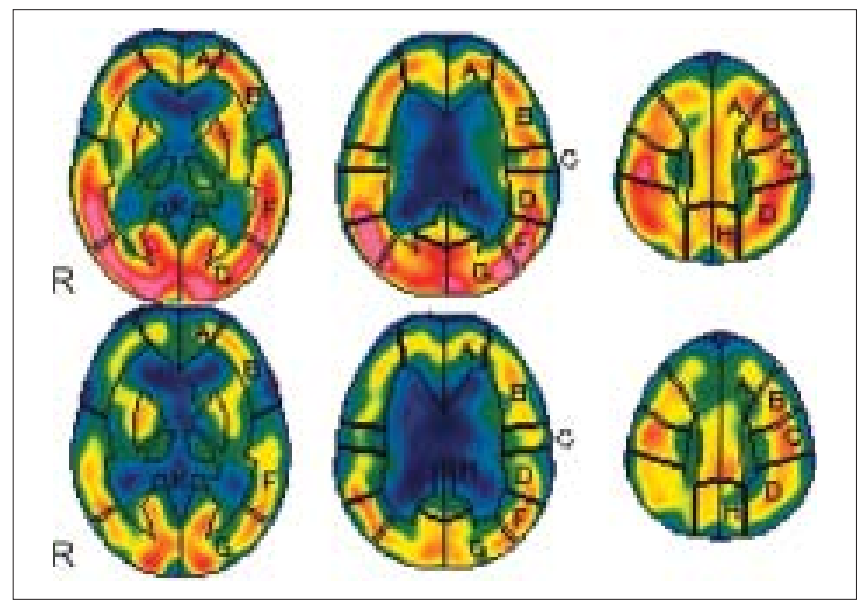

Figure 2: SPECT using ${ }^{99 m}$ Tc-ethyl cysteinate dimer (ECD) demonstrating diffuse hyperperfusion in both hemispheres, especially in the bilateral temporoparietal regions on the second hospital day (top) and relative hypoperfusion on the eighth hospital day (bottom)

on CBF changes in hypoglycemic coma.

Recent reports have suggested that DWI is useful to detect acute brain injury in hypoglycemia as well as in the acute stage of ischemia. ${ }^{[1]}$ MRI lesions previously reported in association with hypoglycemia typically involved the cerebral cortex, basal ganglia, and the hippocampus, mostly with poor prognosis resulting in death or persistent vegetative state. These regions are known to be most vulnerable to hypoglycemic insult. Besides these regions, the middle cerebellar peduncle, internal capsule, corona radiate, substantia nigra, and the splenium of the corpus callosum have been reported to be involved in hypoglycemic encephalopathy. In both cases presented here, ischemia due to arterial occlusion or stenosis was excluded by MR angiography (not displayed) and hyperintensity lesions seen on DWI were consistent with the previously reported regions in hypoglycemia. Accordingly, we determined that the clinical symptoms arose as a result of hypoglycemia. 

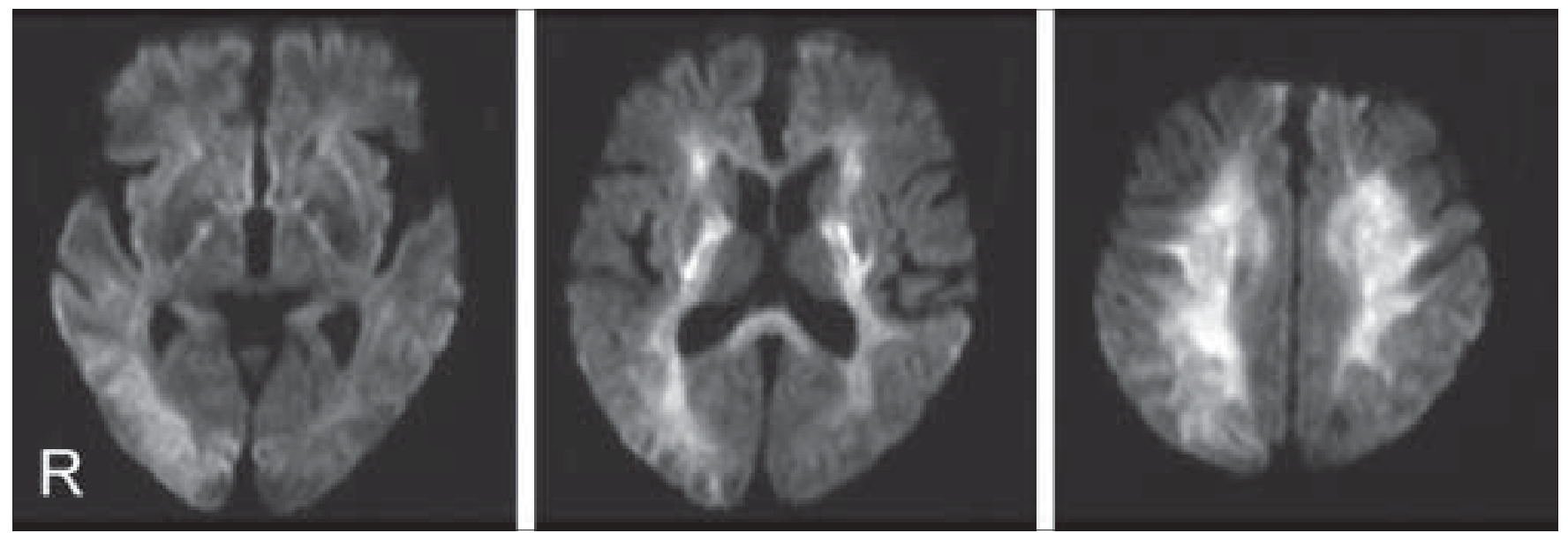

Figure 3: Diffusion-weighted images obtained immediately after admission showing multiple hyperintensity legions in the temporoparietal cortex (more pronounced on the right side), internal capsules, deep white matter at the level of the centrum semiovale, and the splenium of the corpus callosum

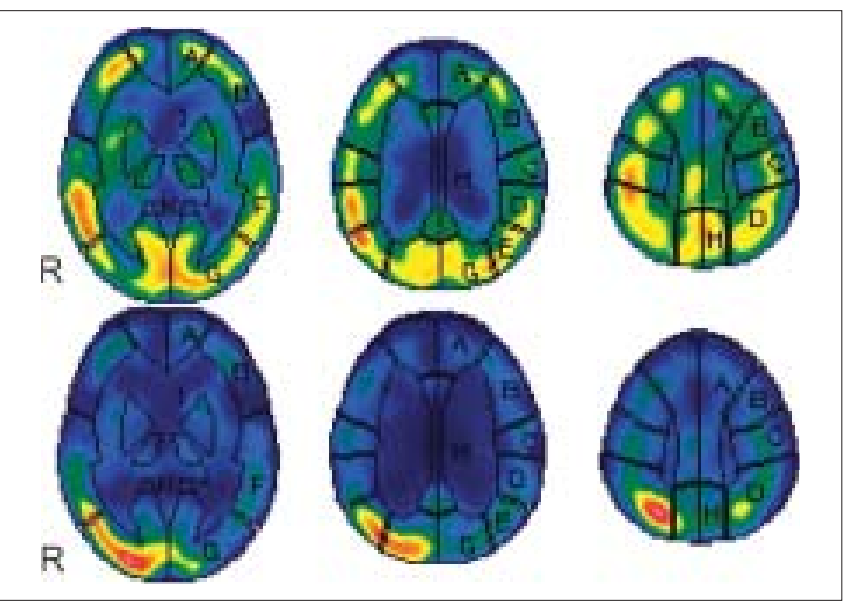

Figure. 4: SPECT using 99mTc-hexamethyl propylene amine oxime (HMPAO) demonstrating relative hypoperfusion except for the right temporoparietal region on the ninth hospital day (bottom) compared to the first one obtained on the third hospital day (top)

From several animal studies, it is known that CBF can increase markedly following both acute severe and moderate hypoglycemia, and is followed by delayed hypoperfusion. ${ }^{[2,3]}$ However, little is known regarding changes of $\mathrm{CBF}$ in humans associated with hypoglycemia. Tallroth et al, analyzed changes in CBF during insulin-induced hypoglycemia in a healthy subject and concluded that moderate hypoglycemia led to a marked increase in CBF, which persisted in the immediate period after normalization of the blood glucose level ${ }^{[4]}$ Cordonnier et al, noted hyperperfusion corresponding to the hyperintensity lesion on DWI in a case of hypoglycemia-induced hemiparesis using perfusion-weighted imaging. ${ }^{[5]}$ In contrast, Shintani et al, reported a case of hypoglycemia-induced hemiparesis in which SPECT using HMPAO showed hypoperfusion in the affected hemisphere ${ }^{[6]}$ Given these clinical data, the influence of hypoglycemia on CBF remains controversial and there is some disagreement as to changes of CBF during hypoglycemia in humans.
Our SPECT data in case 1 seems to corroborate the results of animal studies that CBF could increase in the acute stage of hypoglycemia, although caution is needed when interpreting these SPECT studies due to the lack of controls. Both of our cases also showed relative hypoperfusion in the subacute stage, which might reflect the lower demand of CBF due to diffuse brain injury. We speculate that the local accumulation corresponding to the high intensity lesion on DWI in the second SPECT in case 2 could be due not to hyperemia but hyperfixation of HMPAO, ${ }^{[7]}$ although we cannot fully confirm this conclusion based only on our SPECT data.

Little is known regarding the correlation between SPECT findings and clinical outcome in hypoglycemia, but the degree of relative hypoperfusion is considered to correlate with the severity of hypoglycemia and subsequent neurological sequelae in neonates. ${ }^{[8]} \mathrm{An}$ experimental study which examined the influence of hypoglycemia on regional CBF using SPECT in diabetes patients suggested that $\mathrm{CBF}$ can be increased during hypoglycemia and after normalization of the blood glucose level. ${ }^{[9]}$ Considering these published reports and our results, delayed relative hypoperfusion may indicate poor neurological outcome, but hyperperfusion in the acute stage of hypoglycemia does not necessarily correlate with good neurological outcome.

The underlying mechanism for increasing CBF in hypoglycemic condition is still unclear. Abdul-Rahman et al, found that the increase in CBF was unrelated to hypertension, hypercapnia or hypoxia using an animal model. ${ }^{[2]}$ Bryan et al, mentioned, following a review of the literature, that the increased CBF appeared to be partially attributable to a stress response. ${ }^{[10]}$ Cordonnier et al, mentioned that insulin-induced hypoglycemia could produce cerebral vasodilatation. ${ }^{[5]}$ The SPECT obtained in case 1 demonstrated increased CBF even after glucose correction in the acute stage of hypoglycemia, suggesting 
that other factors besides hypoglycemia itself could have a causal relationship to this phenomenon.

\section{References}

1. Finelli PF. Diffusion-weighted MR in hypoglycemic coma. Neurology 2001;57:933

2. Abdul-Rahman A, Agardh CD, Siesjo BK. Local cerebral blood flow in the rat during severe hypoglycemia, and in the recovery period following glucose injection. Acta Physiol Scand 1980;109:307-14

3. Siesjo BK, Abdul-Rahman A. Delayed hypoperfusion in the cerebral cortex of the rat in the recovery period following severe hypoglycemia. Acta Physiol Scand 1979;106:375-6.

4. Tallroth G, Ryding E, Agardh CD. Regional cerebral blood flow in normal man during insulin-induced hypoglycemia and in the recovery period following glucose infusion. Metabolism 1992;41:717-21.

5. Cordonnier C, Oppenheim C, Lamy C, Meder JF, Mas JL. Serial diffusion and perfusion-weighted MR in transient hypoglycemia.

\section{Neurology 2005;65:175}

6. Shintani S, Tsuruoka S, Shiigai T. Hypoglycaemic hemiplegia: A repeat SPECT study. J Neurol Neurosurg Psychiatry 1993;56:700-1.

7. Sperling B, Lassen NA. Hyperfixation of HMPAO in subacute ischemic stroke leading to spuriously high estimates of cerebral blood flow by SPECT. Stroke 1993;24:193-4.

8. Chiu NT, Huang CC, Chang YC, Lin CH, Yao WJ, Yu CY. Technetium$99 \mathrm{~m}$-HMPAO brain SPECT in neonates with hypoglycemic encephalopathy. J Nucl Med 1998;39:1711-3.

9. Tallroth G, Ryding E, Agardh CD. The influence of hypoglyeaemia on regional cerebral blood flow and cerebral volume in type 1 (insulindependent) diabetes mellitus. Diabetologia 1993;36:530-5.

10. Bryan RM Jr. Cerebral blood flow and energy metabolism during stress. Am J Physiol 1990;259:H269-80.

Accepted on 06-09-2008

Source of Support: Nil, Conflict of Interest: None declared.

\section{Dispatch and return notification by E-mail}

The journal now sends email notification to its members on dispatch of a print issue. The notification is sent to those members who have provided their email address to the association/journal office. The email alerts you about an outdated address and return of issue due to incomplete/incorrect address.

If you wish to receive such email notification, please send your email along with the membership number and full mailing address to the editorial office by email. 